\title{
Evidence of Preliminary Response Preparation From a Divided Attention Task
}

\author{
Jeff Miller \\ University of California, San Diego
}

\begin{abstract}
In a divided attention situation, preliminary response activations produced by stimuli on one channel were revealed through their effects on responses to stimuli on a secondary probe channel. Subjects performed concurrent but independent four-choice reaction-time tasks using the same four response fingers (middle and index fingers on both hands). In the main task, targets were large and small $S s$ and $T s$, and medium-sized $S s$ and $T s$ sometimes appeared as distractors. Targets in the probe task were squares differing in location. A response to a probe square was faster if a distractor letter presented just before it had the same name as the target letter corresponding to that square (i.e., assigned to the same response key) than if the distractor letter had a different name-a result indicating that distractor letters cause partial response preparation. The timecourse of the effect demonstrated that preparation was based on preliminary information about distractor name that was available before distractor size had been determined. The results support models in which response preparation can sometimes begin before stimulus recognition has finished.
\end{abstract}

Recently there has been considerable interest in distinguishing between discrete and continuous models of human information processing (e.g., Meyer, Yantis, Osman, \& Smith, 1984, 1985; Miller, 1982, 1983, 1985b). One important difference between these two types of models concerns the temporal relations among the different mental processes involved in a task. Discrete models require mental processes to operate in strict sequence, so that total reaction time (RT) is the sum of durations of the various processes (e.g., Sternberg, 1969). Continuous models allow later processes to begin before earlier processes have completely finished, resulting in some temporal overlap of successive processes (e.g., Eriksen \& Schultz, 1979; McClelland, 1979). A critical reason for seeking to discriminate between these two types of models, then, is that they have very different implications for the interpretation of RT as a dependent measure (McClelland, 1979).

This article is concerned with the question of whether preliminary information about a stimulus can sometimes be used to begin preparing responses before recognition of that stimulus has finished, as would be allowed by continuous models but prohibited by discrete ones. ${ }^{1}$ The response preparation that hypothetically takes place before stimulus recognition has finished will be referred to as preliminary response preparation. Three paradigms for detecting such preparation have been suggested previously (Miller, 1982, 1983, 1985b), and this article presents

This research was supported by National Institute of Mental Health Grant PHS-MH40733. I would like to thank Carol Fowler, Patricia Haden, Allen Osman, and two anonymous reviewers for helpful comments on an earlier draft of the article, and Lauren Lyon, Eric Metzner, and Lisa Oakes for assistance in collecting the data.

Correspondence concerning this article should be addressed to Jeff Miller, Department of Psychology, C-009, University of CaliforniaSan Diego, La Jolla, California 92093. an experiment that builds on these three paradigms to confirm and extend their results.

The experiment reported in this article had two major goals. One was to defend one of the three previously developed paradigms: the hand-mapping paradigm (Miller, 1982). This paradigm has been criticized (Proctor \& Reeve, 1985; Reeve \& Proctor, 1984, 1985), because it lacks a converging operation to show that the predicted effects of preliminary response preparation actually result from such preparation rather than from some other process. The present experiment was designed to provide such a converging operation.

The second goal was to investigate differences in results of the three previous paradigms. Results from two of the paradigms suggested that preliminary response preparation did occur, but results from the third suggested that it did not. The present experiment was designed to investigate which task properties are most important in determining whether preliminary response preparation occurs. To this end, elements of all three previous paradigms were incorporated in the present experiment. The three previous paradigms (and criticisms of them) will first be reviewed briefly, and then the rationale for the present experiment will be described.

\section{Previous Paradigms for Detecting Preliminary Response Preparation}

Two requirements must be met for a paradigm to indicate whether response preparation can begin before stimulus recognition has finished. First, the stimulus recognition process must

\footnotetext{
${ }^{1}$ There are also intermediate models that allow response preparation to begin before stimulus recognition has finished with some stimuli but not others, as discussed by Miller (1982). Because the present article is concerned solely with the issue of how to diagnose preliminary response preparation, it will not address this theoretical complication.
} 
be drawn out in time, with some stimulus information being recognized relatively quickly (early information) and other stimulus information being recognized relatively slowly (late information).

According to continuous models, the early information acquired by the stimulus recognition process is immediately transmitted to the response preparation process so that the later process can begin before the earlier process has finished. According to discrete models, the early information is not made available to the response preparation process (or not used by it) until the late information has also been recognized, at which time the stimulus recognition process terminates. Note that if all stimulus information were recognized in a punctate manner, not even continuous models would allow temporal overlap of recognition and response processes. Overlap can occur only when early information is available to let decision and response processes begin, and later information is still needed so that recognition processes must continue.

All three previous paradigms fulfilled this requirement by using a stimulus set requiring two unequally difficult discriminations: letter name ( $S$ vs. $T$ ) and size (Miller, 1982, 1983, 1985b). Pilot work had shown that the letter name discrimination was much easier than the size discrimination, so it was assumed that the name of a given letter would be recognized before its size. In terms of this stimulus set, then, the question is whether a letter's name can be used to prepare responses before its size has been determined.

The second requirement is that the paradigm provide a way to detect preliminary response preparation if it occurs. That is, there must be some specific measurable effect(s) on performance to support the hypothesis that postrecognition processing begins before stimulus recognition is finished. The three paradigms used previously were based on three different indices of such response preparation.

Before describing the previous paradigms for measuring response preparation, it is necessary to be more specific about what is meant by response preparation. Because the goal of this research program is to discriminate between discrete and continuous models, the crucial question is whether preliminary information about a stimulus is used by any postrecognition process before stimulus recognition is complete, which would be contrary to discrete models. Obviously, discrete models could be contradicted in many different ways. Perhaps the most extreme example is that preliminary information could cause activation of particular motor effectors, like that demonstrated by Coles, Gratton, Bashore, Eriksen, and Donchin (1985). This example is extreme in that the preliminary perceptual information would have to be processed through all stages of the information processing system before recognition was finished, in order for the effect to appear at a peripheral motor level. Slightly more centrally, preliminary information might be used to specify one or more features of a motor program (Rosenbaum, 1980) without actually producing any effector activation. Even more centrally, preliminary information might simply prime stimulus-response (S-R) translation processes so as to speed their action once full information became available. Though the term response preparation is arguably a misnomer in this third example, evidence of such priming would still count as decisive evidence against discrete models in that it would reflect a post- recognition process using preliminary information to prepare for responding before stimulus recognition had finished.

In this article the term response preparation is used to refer to effects on motor activation or motor program specification, because the observed effects seem to be most easily explained in terms of such processes. It should be kept in mind, however, that the inferences concerning discrete and continuous models are valid even if the effects are actually produced by premotor processes (indeed, even preresponse processes), as long as these are postrecognition processes using preliminary information.

\section{Hand-Mapping Paradigm}

There is evidence that response preparation is more efficient when two response fingers on the same hand are prepared simultaneously than when two response fingers on different hands are prepared simultaneously (cf. Miller, 1982, 1985a). Miller (1982) used a four-choice RT task, and cued subjects in advance that the upcoming test stimulus would require a response with one of two particular fingers (cf. Rosenbaum, 1980). Subjects responded more quickly to the test stimulus if the cue had indicated two response fingers on the same hand than if it had indicated two response fingers on different hands.

Capitalizing on the same-hand preparation advantage, Miller (1982) proposed the hand-mapping paradigm as an index of preliminary response preparation. Small and large $S$ s and $T$ s were presented one at a time, with each stimulus assigned to one of four response keys corresponding to the index and middle fingers of the two hands. Two mapping conditions were compared, varying in the mapping of stimuli to responses. In the same-hand mapping condition, letters with the same name were assigned to response fingers on the same hand. In the differenthand mapping condition, letters with the same name were assigned to response fingers on different hands.

The logic of the hand-mapping paradigm is as follows: If response preparation can begin before size recognition is finished, then responses should be faster with a same-hand mapping than with a different-hand mapping. By the hypothesis of response preparation, a letter's name will be used to prepare responses before its size has been recognized. Thus, two response fingers on the same hand will be prepared in the same-hand mapping condition, and two response fingers on different hands will be prepared in the different-hand mapping condition. Because preparation of two fingers on the same hand is more efficient than preparation of two fingers on different hands, as shown by cuing studies, subjects should be able to prepare responses more efficiently in the same-hand mapping condition. More efficient preparation should lead to faster responses with that mapping than with a different-hand mapping.

Results obtained with the hand-mapping paradigm were consistent with the hypothesis that letter name is used to prepare responses before size is recognized (Miller, 1982). Specifically, responses were about $85 \mathrm{~ms}$ faster with same-hand mappings than with different-hand mappings.

The validity of the hand-mapping paradigm has been criticized by Reeve and Proctor (1984, 1985; Proctor \& Reeve, 1985). First, they questioned the cuing paradigm indicating that two response fingers on the same hand are prepared more efficiently than two response fingers on different hands. In the cu- 
ing experiments conducted by Miller (1982), the hands were adjacent to one another, so that two fingers on the same hand were also on the same side of the response panel. Reeve and Proctor (1984) repeated the cuing experiment including a condition in which subjects overlapped the fingers of the two hands, so that two fingers on the same hand were on different sides of the response panel, and two fingers on the same side of the response panel were on different hands. In this condition, they found faster responses following cuing of two response fingers on the same side rather than on the same hand. Because the cuing effect was tied to response location rather than to finger, they concluded that it was not an indicator of response preparation.

Second, Proctor and Reeve (1985) also repeated the handmapping experiment including a condition with overlapped fingers, and they found that the hand-mapping effect was also tied to locations rather than to fingers. Because effects of $S-R$ compatibility are also tied to response locations rather than to response effectors (e.g., Anzola, Bertoloni, Buchtel, \& Rizzolatti, 1977; but see Klapp, Greim, Mendicino, \& Koenig, 1979), they concluded that the hand-mapping effect probably reflects S-R translation processes rather than response preparation.

Though the results obtained with overlapping response fingers are consistent with an $S-R$ compatibility explanation of the hand-mapping effect, they do not require such an explanation. It is often implicitly assumed that response preparation processes are organized in terms of effectors (e.g., Miller, 1982; Rosenbaum, 1980), but this assumption is not necessary, especially given the broad notion of response preparation appropriate for discriminating between discrete and continuous models. Response preparation could instead be controlled by a system that was more sensitive to response locations than to response effectors. In other words, the location dependence of the handmapping effect does not preclude its being a result of response preparation processes. Likewise, the importance of response location in the cuing paradigm could easily be interpreted as showing that response preparation processes code responses in terms of location, instead of being interpreted as showing that cuing effects do not reflect response preparation. How location and effector specification jointly control response preparation is of interest within models of exactly how such preparation takes place, but it is not critical for the issue of whether such preparation takes place on the basis of preliminary information.

To decide whether the hand-mapping effect reflects response preparation, it is necessary to have a converging operation to test more directly the involvement of response preparation processes in the effect. The present experiment uses the hand-mapping paradigm in conjunction with a secondary task to provide such a converging operation.

\section{Discriminability-by-Hint Interaction Paradigm}

A second way to measure preliminary response preparation is to see how it interacts with the preparation resulting from explicit hints given prior to stimulus onset. Miller (1983) used the same-hand mapping condition of the previous paradigm, with two additional features. First, before stimulus presentation the subject was sometimes given a hint as to which hand would make the response. This hint was designed to allow response preparation before stimulus onset, thereby canceling out any benefit of the hypothesized preliminary response preparation based on early information about stimulus name. Second, difficulty of the size discrimination was varied. The difficulty of the size discrimination controls the amount of opportunity for preliminary response preparation, because response preparation takes place during the interval from recognition of stimulus name to recognition of stimulus size. The longer this interval (i.e., the harder the size discrimination), the more preliminary response preparation should occur.

In this paradigm, preliminary response preparation would produce an interaction between the presence versus absence of a hint and the difficulty of the size discrimination: Presentation of a hint should produce less benefit when the size discrimination is hard than when it is easy. This is so because the difficult size discrimination would allow more time for preliminary response preparation (i.e., response preparation using stimulus name that is carried out before stimulus size has been recognized) than the easy size discrimination. Because more preparation is done after stimulus onset with a difficult size discrimination than with an easy one, there would be less benefit from preparing in advance by using a hint. If more preparation can be done during the size discrimination, there is less to gain from doing it in advance. The predicted underadditive interaction was obtained, supporting the hypothesis that preliminary response preparation does occur. Proctor and Reeve (1986), however, were unable to replicate this underadditive interaction in RT. They did obtain the interaction in error rates, but argued that the spatial rather than anatomical nature of the errors implicated S-R translation processes as the source of the errors.

\section{Two-Choice Divided Attention Paradigm}

Miller (1985b) used a divided attention paradigm as a third test for response preparation. A two-alternative forced-choice task was employed, with one visual and one auditory target assigned to each response. Visual targets were letters varying in identity and size (e.g., small $S$, large $T$ ), and auditory targets were high- and low-pitched tones. On each trial a target was presented on one modality, and a distractor was presented on the other. Visual distractors were letters with the same names as targets but different in size (e.g., medium $S$, large $S$, small $T$, medium $T$ ), and auditory distractors were tones of medium pitch.

The divided attention paradigm allows measurement of response preparation in terms of the effect of a visual distractor on RT to an auditory target. For example, one of the distractor Ss might be presented, followed up to $300 \mathrm{~ms}$ later by a tone target. If a letter name causes response preparation, then a distractor $S$ should cause some preparation of the response associated with the target small $S$. This preparation should reduce RT when the tone target requires that the prepared response actually be made, but it should increase RT when the tone target requires the opposite response. Thus, if letter name is used for response preparation, there should be an effect of the consistency of the distractor name with the response required to the tone (cf. Eriksen \& Schultz, 1979).

Continuous models predict that such a consistency effect 
should occur, because response preparation would take place during the time interval after the name had been recognized but before the size had been recognized. Discrete models do not directly predict a consistency effect, but they can be reconciled with it if response preparation is assumed to occur after both name and size have been recognized. Thus, unambiguous support for continuous models requires evidence that the consistency effect arises from processing that takes place before size recognition is complete.

Results obtained with this divided attention paradigm were most compatible with discrete models. There was a consistency effect for medium-sized distractor letters, indicating that visual distractors can produce enough response preparation to influence responses to auditory targets. However, large and small distractors did not produce any consistency effect. If the consistency effect were produced during the interval before size recognition was complete, then distractors of all sizes ought to produce the same consistency effect, because the distractors differing only in size are not distinguishable to the system at that point. Thus, this pattern of results suggests that response preparation does not begin until recognition of the visual distractor is complete.

It seems likely that there was no consistency effect for large and small distractors because the two attributes of the distractor-name and size-produced approximately equal response activation. These two types of activation would cancel each other out, because they always favored opposite responses in this paradigm. With one attribute activating each response, there would be no advantage for tones requiring the response consistent with distractor name as opposed to those requiring the response consistent with distractor size. The explanation that distractor name and size produced approximately equal response activation suggests that response processes did not start until both attributes of the distractor had been recognized, as assumed by discrete models. If response processes had started as soon as the early information was available (i.e., distractor name), then the response associated with the distractor name should have received greater activation.

\section{Summary}

In the hand-mapping and discriminability-by-hint paradigms, subjects chose among four alternative responses made with the index and middle fingers of the two hands (Miller, 1982, 1983). The results of both paradigms were consistent with what would be expected if letter name were used to prepare responses before size was recognized, though it would have been desirable to have further evidence that response preparation was actually responsible for the obtained effects. In the divided attention paradigm there were only two alternative responses made with the two index fingers (Miller, 1985b), and the results suggested that letter name was not used for preliminary response preparation.

\section{Experiment}

The purposes of the present experiment were to seek additional evidence that the hand-mapping effect observed by Miller (1982) actually reflects response preparation and to start trying to identify what aspects of the two-choice divided attention paradigm were responsible for the lack of preliminary response preparation in that task. Response preparation was measured by using a combination of the hand-mapping and divided attention paradigms.

Subjects performed two four-choice RT tasks concurrently. In the main task, single letters were presented at fixation. ${ }^{2}$ Subjects responded to large and small $S$ s and $T s$ that were targets for this task, but did not respond to medium-sized distractor $S \mathbf{s}$ and $T$ s. Four response keys were arrayed from left to right in front of the subject, and the index and middle fingers of the two hands rested on these response keys in the most natural fashion. Mappings of target letters to response keys were varied across subjects.

In the secondary task, stimuli were bright squares appearing at one of four locations in the lower half of the visual field. If one of the four squares brightened, the subject was to press the spatially corresponding response key as quickly as possible.

In this paradigm, three effects can be predicted from the hypothesis that preliminary response preparation takes place before distractor size recognition is finished. First, as in the divided attention paradigm of Miller (1985b), there should be an effect of the consistency of the distractor's name with the response required by a square. On the trials of interest, subjects were first presented with a medium-sized distractor letter, and presented soon after with a bright square (stimulus onset asynchrony, $\mathrm{SOA}=100-2,000 \mathrm{~ms}$ ). Because letter name is more discriminable than letter size, there should be a brief interval during which subjects know what letter name was presented but not what size the letter is (or whether it was a target or distractor). Suppose preliminary response preparation takes place during the interval when name has been recognized but size has not. Then responses corresponding to that letter name should become activated before the subject realizes that it is a distractor. If a square appears during this time, the response to the square should be faster if the square requires one of the responses already activated by the distractor name than if it requires one of the unactivated responses.

Second, the hand-mapping effect of Miller (1982) should also be obtained in this paradigm. The mapping of target letters to response keys was varied across subjects, with half of the subjects in the same-hand mapping condition and half in the different-hand condition. If letter name is used to prepare responses before size has been recognized and if preparation of two response fingers on the same hand is more efficient than preparation of two response fingers on different hands, then responses should be faster for subjects with same-hand mappings than for subjects with different-hand mappings.

Third, the consistency effect should be larger with same-hand mappings than different-hand mappings. This interaction of consistency and mapping is predicted from the assumption that preparation of two response fingers on the same hand is more efficient than preparation of two response fingers on different

\footnotetext{
${ }^{2}$ The terms main and secondary task are used bere to denote the importance of the tasks for the hypotheses being tested. From the point of view of the subject, the two tasks received equal emphasis by the experimenter.
} 
hands. ${ }^{3}$ If the consistency effect does reflect preliminary response preparation, then this effect should be larger in the condition in which more effective response preparation takes place (same hand).

According to discrete models, there should be no response preparation based on early information about a distractor's name. There might still be a consistency effect, however, if some response preparation took place after distractor recognition was complete, as found by Miller (1985b). On this view, the distractor would act like a hint to isolate or suggest two particular responses, as in the cuing experiments of Miller (1982). Two further variables were manipulated to test the hypothesis that the expected consistency effect resulted from response preparation taking place before the distractor stimulus had been fully recognized.

First, the stimulus onset asynchrony (SOA) between the onset of the visual distractor and the onset of the bright square was varied from 100 to $2,000 \mathrm{~ms}$ to see whether the consistency effect is caused by a process that changes nonmonotonically during the trial. If the consistency effect is produced by activation resulting from preliminary information about the stimulus, then it should first increase and then decrease as SOA is lengthened. At the earliest SOA the consistency effect should be small because the distractor will not have had time to cause any response preparation by the time the square appears. The consistency effect should build during the time when the name of the distractor has been identified but its size has not, and this should lead to a maximal consistency effect shortly after distractor presentation. ${ }^{4}$ Once distractor size has also been identified, though, response prepiatition processes should cease, because they will have the information that the stimulus is a distractor. At this point the consistency effect should stop increasing and start to decrease, assuming that response preparation will wane once the available stimulus information no longer supports any responses. Such a waxing and waning of the consistency effect would be consistent with the hypothesis that it is preliminary rather than full knowledge about the stimulus which causes preparation.

How should the consistency effect change with SOA if it were instead produced by processes occurring after discrete recognition of the distractor? Because cuing effects are monotonically increasing over the first few seconds of cue presentation (Reeve \& Proctor, 1984), a cuelike effect of the distractor should also produce a monotonic increase in the consistency effect. The effect would build with initial increases in SOA, because these increases provide more time for the distractor to be recognized and for the cuing processes to be carried out. The effect should not then decrease within the next $2 \mathrm{~s}$, however, because no new information about the stimulus becomes available during that time.

Second, the difficulty of the size discrimination was varied. When the size discrimination is easier, there is less time between recognition of stimulus name and recognition of stimulus size; thus there is less opportunity for preliminary response preparation. Thus, the three predicted effects of preliminary response preparation-the consistency effect, the mapping effect, and the Consistency $\times$ Mapping interaction-should all be smaller when the size discrimination is easy than when it is difficult.

If the consistency effect is produced after full distractor recog- nition, however, it seems unlikely that the difficulty of the size discrimination should have any influence on the consistency effect. Regardless of size difficulty, the consistency effect would be produced by a process privy to the knowledge that the stimulus was a distractor. The name-based consistency effect should therefore probably not depend on whether the distractor was slightly or very different from targets in size.

\section{Method}

Subjects and apparatus. One hundred twenty-eight undergraduates at the University of California, San Diego, served as subjects in partial fulfillment of a course requirement. Each subject was tested in a single session lasting about $\mathbf{4 5} \mathrm{min}$. Half of the subjects were assigned to the same-hand mapping condition, and half to the different-hand condition. Within each of these two groups, half had the easy size discrimination, and half had the hard size discrimination.

Stimuli were presented and responses and their latencies were recorded by an Apple II+ microcomputer, enabling generation of a new random stimulus order for each subject and block. The outside four keys on the bottom row of the computer keyboard ( $Z, X$, period, and slash) were used as response keys, and subjects responded by pressing these keys with the index and middle fingers of the left and right hands.

Stimuli. Letter stimuli were $S_{\mathrm{s}}$ and $T \mathrm{~s}$, presented as light green figures on the dark background of an Amdek monitor, and they were centered at the point of fixation. Small, medium, and large letters were approximately $19 \mathrm{~mm}, 22.5 \mathrm{~mm}$, and $28.5 \mathrm{~mm}$ high, respectively, for the hard size discrimination. For the easy size discrimination, the small letters were the same size as the small ones in the hard size discrimination; the medium letters were the same size as the large ones in the hard size discrimination; and the large ones were about $47 \mathrm{~mm}$ high. Letter widths were about $65 \%$ of their heights. The four squares were approximately $12.5 \mathrm{~mm}$ on a side, and they were displayed $32 \mathrm{~mm}$ below the point of fixation. The two innermost squares were about $12.5 \mathrm{~mm}$ from fixation, and the two outermost squares were about $41 \mathrm{~mm}$ from fixation (measured to inside edge of each square). The stimulus display was viewed from a distance of about $60 \mathrm{~cm}$.

Procedure. Each subject completed four blocks of trials. The first block was a practice block of 60 trials to familiarize subjects with the task of responding to squares. Each trial began with the onset of a fixation point for $1 \mathrm{~s}$. Subjects were instructed to fixate at this point and not to move their eyes when squares came on below the fixation point. About $\mathbf{5 0 0} \mathrm{ms}$ after the offset of the fixation point, outlines of the four squares appeared. After a delay of $100,300,500,1,000$, or $2,000 \mathrm{~ms}$, one of these outline squares filled in with the bright green of the display monitor, and this display remained until the subject responded. Accuracy feedback was given after each response $(600 \mathrm{~ms}$ for correct responses and $1.2 \mathrm{~s}$ for errors), and there was a delay of approximately $1 \mathrm{~s}$ from the offset of the accuracy feedback to the onset of the plus sign beginning the next trial. This block began with 20 randomly selected warm-up trials, and then each of the four bright squares was presented twice at each of the five SOAs.

\footnotetext{
${ }^{3}$ For convenience, "same hand" will be used in place of "same hand or same side" throughout this article, because effector and location are confounded in the present experiment. As discussed above, the question of which aspect controls response preparation is secondary to the question of whether such preparation occurs at all.

${ }^{4}$ Because it may take a little time for stimulus information to be made available to response preparation processes, these processes may lag somewhat behind stimulus identification processes. Thus, the absolute time at which the consistency effect is maximal need not correspond to the absolute time at which size information becomes available.
} 
The second block provided 80 trials of practice at the task of responding to letters. Before the block began, subjects were shown the four target letters (large and small $S$ and $T$, and they were taught the assignment of letters to response keys. They were instructed to press the corresponding key as quickly as possible if any of these four letters appeared. Subjects were also told that two distractor letters-a medium-sized $S$ and a medium-sized $T$-would sometimes be presented. Subjects were instructed not to press any response key when these letters appeared.

The second block also began with 20 randomly selected warm-up trials, and then each of the six stimulus letters was presented 10 times. The structure of a trial was the same as in the first block, except that a letter was presented simultaneously with the row of four outline squares, and no squares ever brightened. Target letters remained on the screen until subjects responded, and distractor letters remained on the screen for 2 s or until a response was made, whichever came first.

The four-choice divided attention task was introduced for the third and fourth blocks. Subjects were instructed that this block was a combination of what they had done in the first two blocks: On some trials they would get a large or small $S$ or $T$, and they were to press the corresponding response key as quickly as possible when they did. On the other trials they would get a medium-sized $S$ or $T$, and when this happened, one of the four squares would brighten to indicate which response they should make.

The third block began with 20 randomly selected warm-up trials, followed by 160 test trials. Each of the four test letters was presented on 20 of the test trials, and each of the two distractor letters was presented on $\mathbf{4 0}$ of the test trials. Each distractor letter was presented twice with each of the 20 combinations of five SOAs $(100,300,500,1,000,2,000$ ms) and four responses (i.e., four different brightened squaies). A test letter and a bright square were never presented on the same trial. The fourth block was identical to the third, except that only four warm-up trials were used and a new random order of stimuli was generated.

Across subjects all 24 possible mappings of letter stimuli to response keys were used. Each of the 8 same-hand mappings was used for 4 subjects, and each of the 16 different-hand mappings was used for 2 subjects within both size discrimination conditions.

\section{Results and Discussion}

For each subject, block, and condition, the percentage of correct responses (PC) and median RT for correct responses were computed. Parallel analyses were performed with the two dependent variables, though the primary interest was in the RT measure. Significant effects on PC are reported as applicable to the interpretation of the RT data.

Preliminary analyses examined performance in the two focused-attention blocks that were run as practice for the divided attention task. In the first block, responses to squares averaged $514 \mathrm{~ms}$ in duration, with an overall accuracy of $98.3 \%$. RTs to squares varied significantly as a function of SOA, $F(4,496)=$ $8.3, M S_{c}=1,953, p<.01$, with averages of $519,496,509,523$, and $522 \mathrm{~ms}$ across the five SOAs. Accuracy atso varied with SOA, $F(4,496)=3.6, M S_{e}=19.91, p<.01$, with averages of $98.8 \%, 98.4 \%, 97.6 \%, 97.6 \%$, and $99.2 \%$ across the five SOAs. The random assignment of subjects to mapping and discriminability conditions was effective, as indicated by a lack of significant main effects or interactions of these factors in the analyses of responses to squares.

In the second focused-attention block, responses to letters averaged $957 \mathrm{~ms}$, with an overall accuracy of $96.2 \%$. The effect of discriminability was only $40 \mathrm{~ms}$, and was not significant in this between-subjects comparison, $F(1,124)=2.15, p>.10$. Re-

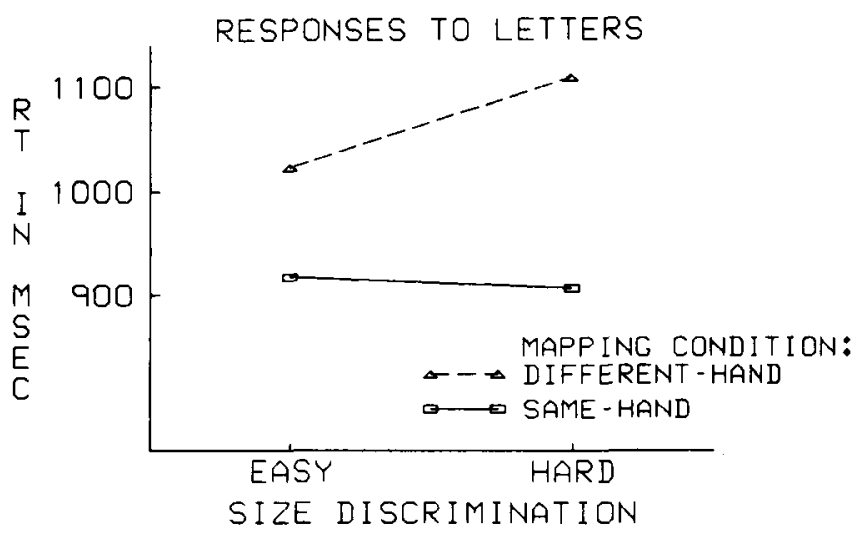

Figure I. Reaction time (RT, in milliseconds) to target letters as a function of mapping condition and discriminability (divided attention task).

sponses were $180 \mathrm{~ms}$ faster, $F(1,124)=44, M S_{\mathrm{e}}=23,210, p<$ .01 , and $1.8 \%$ more accurate, $F(1,124)=7, M S_{\mathrm{e}}=15.98$, $p<.01$, with a same-hand mapping than with a different-hand mapping. This mapping effect is larger than is usually obtained, and the absolute times are somewhat longer. Both discrepancies probably result from the relatively small amount of practice reflected in this condition. No interaction of discriminability and mapping was obtained. The percentage of false alarms (i.e., responses to distractor letters) was $8.5 \%$ overall, with $12.9 \%$ in the hard discriminability condition and $4.1 \%$ in the easy discriminability condition, $F(1,124)=11, M S_{\mathrm{\varepsilon}}=222, p<.01$. False alarms were also more common with a different-hand mapping (11.3\%) than with a same-hand mapping $(5.6 \%), F(1,124)=$ $4.68, p<.05$. Almost all of the false alarms $(96.8 \%)$ were made by pressing one of the two response keys assigned to letters with the same name as the medium-sized distractor letter.

Responses to target letters. Figure 1 shows average RT to target letters in the divided attention task, as a function of mapping condition and size discriminability. Because both of these factors were between subjects, statistical reliability was evaluated with an analysis of covariance, using median RT from the first practice block (responding to squares) as the covariate. Responses were $155 \mathrm{~ms}$ faster in the same-hand mapping condition than in the different-hand mapping condition, $F(1,123)=$ $29, p<.01$, and $37 \mathrm{~ms}$ faster with an easy size discrimination than with a difficult one, $F(1,123)=3.1, p<.10$. The interaction of these two factors, shown in Figure 1, was also significant, $F(1,123)=4.2, p<.05$, as was the covariate, $F(1,123)=34$, $p<.01$. Simple main effects tests of the interaction (Keppel, 1973) showed that the discriminability effect was significant for the different-hand mapping group $(p<.025)$ but not the samehand mapping group ( $p>.25$ ). For all these analyses, $M S_{\mathrm{c}}$ was 19,992. The PCs for the four conditions in Figure 1 were (clockwise from upper right) $95.5,96.0,95.7$, and 96.7 ; no significant effects were obtained on this dependent variable.

The finding of a significant mapping effect on RT to target letters fulfills one prediction following from the hypothesis of preliminary response preparation and extends the results of Miller (1982) to a divided attention task. In fact, the mapping effect was somewhat larger under the divided attention conditions of the present experiment than in the original single-stim- 
ulus task, probably because the task takes longer overall with attention divided.

The fact that the mapping effect is larger with the harder size discrimination also supports the hypothesis of preliminary response preparation. This interaction suggests that the mapping effect is produced during the interval when letter name has been recognized but size has not. Increasing the duration of this interval should increase the opportunity for response preparation, which should in turn increase the mapping effect.

It is particularly interesting that no discriminability effect at all was obtained with the same-hand mapping. Because a small effect was obtained with the different-hand mapping, it appears that discriminability did have an effect on perceptual processing time. Why wasn't this effect reflected in total RT with a same-hand mapping? One plausible explanation, entirely consistent with preliminary response preparation, is that an additional process operated in parallel with the extra processing needed for the difficult size discrimination. This additional process could have facilitated responding enough to cancel out the extra perceptual processing time required for the difficult discrimination. Response preparation is an obvious candidate to be the additional process required by such an explanation.

Responses to squares. Figure 2 shows RT to squares as a function of the consistency of the name of the visual distractor and the mapping condition. As predicted, the consistency effect was larger with the same-hand mappings ( $22 \mathrm{~ms}$ ) than with the different-hand mappings $(6 \mathrm{~ms})$. Statistical analysis indicated that the 14-ms main effect of consistency was highly reliable, $F(1,124)=26, M S_{\mathrm{\varepsilon}}=4,860, p<.01$, as was the interaction of consistency with mapping condition, $F(1,124)=7.22, M S_{8}=$ $3,190, p<.01$. The 17 -ms main effect of mapping-a betweensubjects effect-was not reliable, $F(1,124)<1$. Indeed, the hypothesis of preliminary response preparation does not predict any effect of the mapping of target letters to response keys on RT to squares, averaged across distractor consistency. The PCs corresponding to the four conditions in Figure 2 were (clockwise from upper right) 97.3, 97.2, 98.2, and 97.9; they showed only a significant main effect of consistency, $F(1,124)=14$, $M S_{e}=30, p<.01$.

The main effect of consistency indicates that visual distractors do influence responses to squares. A distractor name activates the responses with which it is consistent, facilitating responses to squares requiring those responses and/or inhibiting responses to squares requiring competing responses. Because this consistency effect extends from one task to another, it seems likely that the effect is based on response processes rather than $S-R$ translation processes. There seems to be no good reason why a letter distractor would influence $S-R$ translation processes involving squares. Residual response activation, however, would certainly be expected to have such a cross-task effect.

Given that the consistency effect reflects response activation, the interaction of mapping and consistency is evidence that the mapping effect is at least partially a reflection of response activation processes. Thus, it appears that response activation is more effective with a same-hand mapping than with a differenthand mapping, as assumed by Miller (1982). This conclusion, in turn, supports the contention that the mapping effect is a valid measure of response preparation.

To test the hypothesis that at least some of the consistency

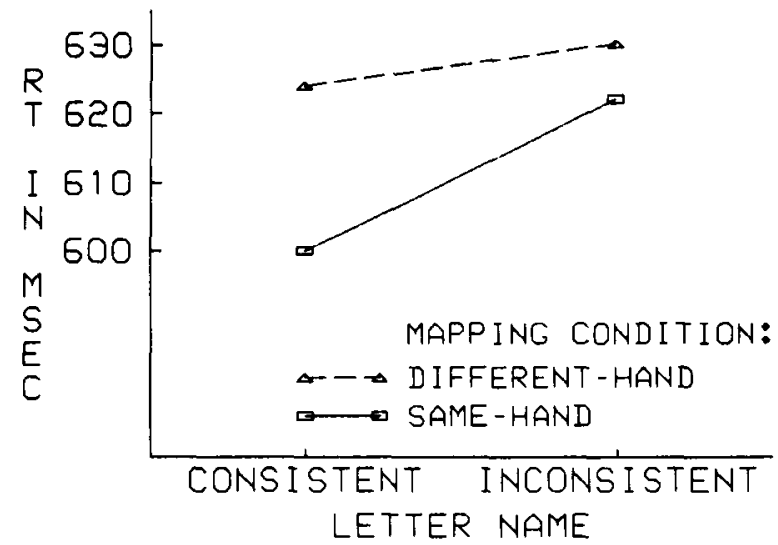

Figure 2. Reaction time (RT, in milliseconds) to squares as a function of the consistency of the name of the visual distractor and the mapping condition (divided attention task).

effect arises before the visual distractor has been fully identified (i.e., results from preliminary response preparation), it is necessary to examine the consistency effect as a function of SOA and size discriminability. Table 1 shows the interaction of SOA and consistency, and it is apparent that the average consistency effect first waxes and then wanes with increasing SOA. This interaction merely approached significance in the omnibus $F$ test, $F(4$, 496) $=2.04, M S_{e}=3,458, p<.10$, but this test is very conservative because it ignores the systematic nature of the predicted interaction. To test for the predicted nonmonotonic interaction, a quadratic trend test was computed for the function relating the consistency effect to SOA (Keppel, 1973). This trend was statistically significant, $F(1,496)=6.65, p<.01$, indicating that the waxing and waning in the means is not attributable to chance.

Planned comparisons were also performed to evaluate the consistency effect at each individual SOA, with results indicated in the table. These comparisons reinforce the conclusion that the consistency effect waxes and then wanes with increases in SOA. A similar effect was apparent in the analyses of PCs. Significant consistency effects of about $1 \%$ were obtained at SOAs of 300 and $500 \mathrm{~ms}$ ( $p s<.01$ and .05 , respectively), but no significant effects were obtained at any of the other SOAs (all $p s>.10$ ).

The waxing and waning of the consistency effect with increasing SOA supports the hypothesis that the consistency effect results from preliminary response preparation. If the effect were caused by processes occurring after the distractor had been fully identified, one would expect that the effects would simply grow with the time until square onset, as do cuing effects. However, if the effect were caused by a process that changed nonmonotonically during the trial, as response preparation processes would change based on inputs first about stimulus name and then about stimulus size, then a waxing and waning pattern would be obtained.

If the consistency effect reflects response preparation taking place before size recognition is complete, then it is also expected that this effect would be larger with a hard size discrimination than with an easy one. The data indicate an effect in the ex- 
Table 1

Divided Attention Task: Reaction Time (Percentage Correct, PC) to Squares as a Function of Stimulus Onset Asynchrony (SOA) and Distractor Name Consistency

\begin{tabular}{|c|c|c|c|c|c|}
\hline \multirow[b]{3}{*}{ SOA (in ms) } & \multicolumn{4}{|c|}{ Distractor } & \multirow[b]{3}{*}{ Consistency effect } \\
\hline & \multicolumn{2}{|c|}{ Consistent } & \multicolumn{2}{|c|}{ Inconsistent } & \\
\hline & $R T$ & $P C$ & $R T$ & $P C$ & \\
\hline $\begin{array}{r}100 \\
300 \\
500 \\
1,000 \\
2,000\end{array}$ & $\begin{array}{l}703 \\
636 \\
602 \\
544 \\
574\end{array}$ & $\begin{array}{l}99.1 \\
99.3 \\
99.1 \\
97.6 \\
95.3\end{array}$ & $\begin{array}{l}714 \\
656 \\
623 \\
559 \\
577\end{array}$ & $\begin{array}{l}98.7 \\
98.1 \\
98.1 \\
96.9 \\
94.2\end{array}$ & $\begin{array}{l}11^{*} \\
20^{* * *} \\
21^{* * *} \\
15^{* *} \\
3\end{array}$ \\
\hline
\end{tabular}

$* p<.10 . * p<.05 * * * p<.001$.

pected direction ( $18 \mathrm{~ms}$ vs. $10 \mathrm{~ms}$ ), but this effect did not attain statistical significance, collapsing across SOAs in the overall analysis, $F(1,124)=2.55, p>10, M S_{e}=4,860$. However, it is an extremely conservative test of the interaction to collapse across SOAs, because the interaction is expected only at medium to moderately long SOAs. At shorter SOAs, the consistency effect should not depend on size discriminability, because neither easy nor hard size discriminations would have finished. At the longest SOA, because there is no consistency effect, size discriminability cannot influence it. A planned comparison was performed including only SOAs of 500 and $1,000 \mathrm{~ms}$, for which size discriminability would be expected to influence the consistency effect. As predicted, this analysis resulted in a significant interaction of consistency and discriminability, $\boldsymbol{F}(1$, 124) $=4.73, p<.05, M S_{e}=5,088$, with a consistency effect of $28 \mathrm{~ms}$ for hard size discriminations and $9 \mathrm{~ms}$ for easy ones.

One can make a variety of even more detailed predictions regarding the consistency effect as a joint function of mapping size discriminability, and SOA. For example, the consistency effect should be largest with a hard size discrimination, samehand mapping, and SOA of $500 \mathrm{~ms}$, because that combination produces optimal conditions on all factors for a consistency effect. Figure 3 shows the consistency effect as a joint function of all potentially relevant factors: size discriminability, mapping condition, and SOA. Though the four-factor interaction is not significant because of the low power of such a test, the waxing and waning of the consistency effect in different conditions is generally quite consistent with the predictions of the model.

Two other significant interactions involving the mapping effect were obtained in the overall analysis of variance on RT to squares, and both are shown in Table 2 . As is apparent in the right-most column, there was a significant tendency for the overall mapping effect to increase with increasing SOA, $F(4$, 496) $=3.81, p<.01, M S_{e}=6,892$. Furthermore, this tendency was more pronounced when the size discrimination was hard than when it was easy, $F(4,496)=3.08, p<.05, M S_{c}=6,892$. Inspection of the table indicates that at SOAs of 100 and 300 ms the mapping of letter targets to responses has little or no influence on responses to squares. At longer SOAs, however, subjects were faster to respond to squares if they had a samehand mapping of target letters than if they had a different-hand

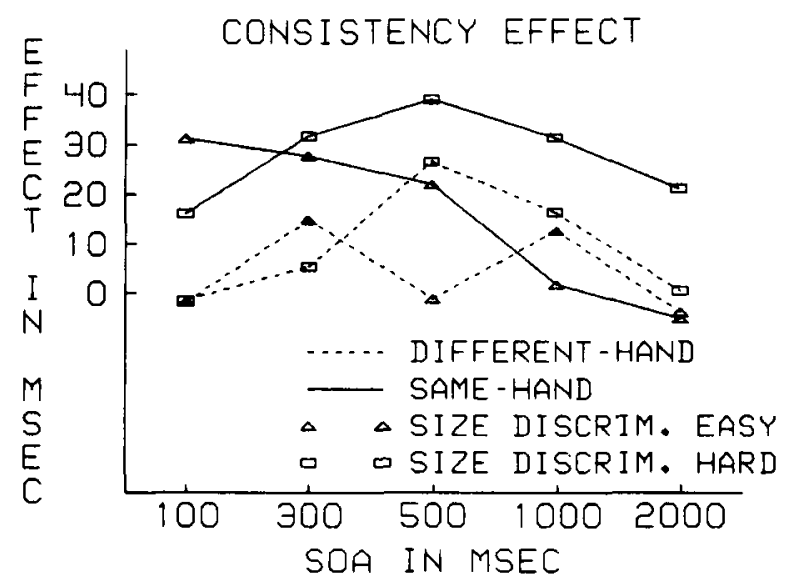

Figure 3. Consistency effect on reaction time (in milliseconds) to squares as a function of size discriminability, mapping condition, and stimulus onset asynchrony (SOA, in milliseconds; divided attention task).

mapping, especially if the size discrimination was difficult. This can be explained as a general difficulty effect of same- versus different-hand response preparation. That is, response preparation may not only be more productive with a same-hand mapping (i.e., producing a larger consistency effect) but may also produce less competitive interference with other processes going on at the same time. When the size discrimination is hard and significant response preparation takes place, then samehand preparation would interfere less than different-hand preparation with the concurrent task of monitoring for squares. Such a generalized difficulty of different-hand preparation would explain the overall effect of mapping at later SOAs as well as the smaller consistency effect for these mappings. In fact, the generalized effect might persist at larger SOAs than the consistency effect for a number of reasons. For example, there may be a resetting process that eliminates response preparation after size recognition has indicated that the visual stimulus is a distractor. This resetting process may be more difficult if two fingers on different hands were prepared than if two fingers on the same hand were prepared.

\section{General Discussion}

\section{Effects of Response Preparation}

When subjects must respond to targets defined in terms of both letter name and size, distractors with the same names as

Table 2

Mapping Effect (in Milliseconds) as a Function of Stimulus Onset Asynchrony (SOA) and Size Discriminability

\begin{tabular}{cccc}
\hline & \multicolumn{2}{c}{ Size discrimination } & \\
\cline { 2 - 3 } SOA & Hard & Easy & Overall \\
\hline 100 & 1 & 13 & 6 \\
300 & -4 & 2 & -1 \\
500 & 24 & 9 & 17 \\
1,000 & 53 & 13 & 33 \\
2,000 & 47 & 7 & 26 \\
\hline
\end{tabular}


targets activate the response fingers to which those targets are assigned. This activation can be measured through its effects on responses to a set of target squares varying in location, to which subjects respond with the same fingers. Specifically, the response to a target square is faster when the distractor has the same name as the target letter assigned to that square's response finger than when the distractor has the opposite name. This consistency effect is likely to result from explicit activation of responses, possibly even to the level of effector activation, because it is an effect of a distractor from one channel on the response to a semantically unrelated target on another channel. Furthermore, there is strong evidence that a similar consistency effect in two-choice focused attention tasks results from effector activation (Coles et al., 1985).

The finding that the consistency effect was larger for samehand mappings than for different-hand mappings provides support for two key conclusions of Miller (1982). First, this finding supports the conclusion that response preparation is more efficient for two response fingers on the same hand than for two fingers on different hands, because more efficient preparation would lead to a larger consistency effect. This conclusion had been supported by results of cuing studies, in which cued preparation of two fingers on the same hand led to faster responses than cued preparation of two fingers on different hands. Though Reeve and Proctor (1984; Proctor \& Reeve, 1985) have noted that response location is more important than response finger in determining cuing effects, the interaction with consistency supports the hypothesis that this is a response preparation effect, even if it does depend on response location rather than on response finger. Thus, the overall pattern of results supports the hypothesis of a response preparation system that is at least as sensitive to response locations as to effectors.

Second, the interaction of mapping and consistency supports the claim that the mapping effect is at least partly due to response preparation. Miller (1982) presented a number of arguments against the hypothesis that the mapping effect was due to S-R translation but had no direct evidence that it was a consequence of response processes. The present interaction constitutes such evidence, because the consistency effect is an index of response preparation. Because the consistency effect is larger with same-hand than different-hand mappings, it is clear that more response preparation is accomplished in the former mapping condition.

\section{Is Response Preparation Based on Preliminary Information?}

One dispute between discrete and continuous models of information processing hinges on the question of whether postrecognition processes can begin before stimulus recognition finishes. To address this debate, it is not enough merely to establish an effect of response preparation. One must also determine whether the response preparation resulted from preliminary information obtained before recognition of the distractor was complete or from full information available after distractor recognition was finished.

Two experimental manipulations were included in this experiment to try to determine whether the consistency effect arose at least partly from processing taking place before recognition of distractor size was complete. One was the SOA between distractor onset and onset of the square target. If the consistency effect is produced mainly before size recognition is complete, then the effect ought to first increase and then decrease as SOA increases, just as it does. The initial increase (SOAs $=100-500$ $\mathrm{ms}$ ) would result from the buildup of response preparation after distractor name had been recognized; prior to this recognition, no consistency effect can be produced. The subsequent decrease (SOAs = 1 and $2 \mathrm{~s}$ ) is expected, because, after size recognition is complete, the known distractor status of the stimulus would terminate response preparation. Of course, it might take some time for previously established preparation to decay to the point where it would no longer have any consistency effect. This would explain the existence of a residual consistency effect at an SOA of $1 \mathrm{~s}$, an SOA at which size recognition would surely be complete.

The results of the size discriminability manipulation also support the idea that preliminary response preparation is at least partly responsible for the consistency effect. At medium to moderately long SOAs, preliminary response preparation was predicted to produce a larger consistency effect when the size discrimination was hard than when it was easy, because the former would allow more time for preliminary preparation to occur. This prediction was confirmed for SOAs of 500 and $1,000 \mathrm{~ms}$.

It should be emphasized that the dependence of the consistency effect on size is even more strongly indicative of processing based on preliminary information than is the dependence of the hand-mapping effect on size (Miller, 1982). The dependence of the hand-mapping effect on size could be attributed to a change in the salience of the codes involved in the S-R mapping (e.g., Proctor \& Reeve, 1985). Though it is ad hoc, one could argue that the dominance of the name code over the size code increases with the difficulty of the size discrimination. Such an argument is more difficult to make when the size code identifies the stimulus as a distractor, however, as it does for all stimuli involved in producing the consistency effect. To postrecognition processes, the salience of the name of a medium-sized distractor would seem to be independent of the difficulty of the size discrimination. Because only the name code is even potentially relevant to the response (i.e., belongs to the target set), it would seem to be fully dominant over the code for a distractor size, regardless of the difficulty of the size discrimination. That is, making the distractors more similar to the relevant stimuli along a continuous dimension like size would not seem to have any effect on how a distractor is processed after its recognition is complete.

\section{Under What Conditions Does Preliminary Response Preparation Occur?}

This experiment helps to delineate further the conditions under which preliminary response preparation can occur, thereby helping to clarify the source of the previous discrepancy between results of the two-choice divided attention paradigm as opposed to the hand-mapping and discriminability-by-hint paradigms. Because evidence of preliminary response preparation was obtained in this experiment, several potential explanations of the discrepancy can be ruled out. For example, the two- 
choice divided attention paradigm required subjects to divide attention and to ignore distractors with the same names as targets. Neither of these requirements was present in the handmapping or discriminability-by-hint paradigms, and either might have caused the recognition process to hold early information instead of making it available to later processes for preliminary response preparation. Both requirements were also present in the four-choice divided attention task, though, indicating that neither is responsible for the lack of preliminary response preparation in the two-choice task.

Alternatively, early information could have been made available to later processes in the two-choice divided attention task but still not used to prepare responses. Perhaps the system prepares responses only when early information is completely definitive in ruling out some response alternatives. In the handmapping paradigm early information is definitive in this way, because the eventual response is always consistent with the early name information. In the divided attention paradigm, however, early information is not definitive, because either response is possible if the early information has been provided by a distractor letter. Again, this explanation can be ruled out by the results of the four-choice divided attention paradigm, because preliminary stimulus information was not completely definitive.

At this point, the most obvious difference between paradigms in which response preparation has been found and those in which it has not is that the former are four-choice paradigms and the latter are two-choice. In the former, early information would partially activate two responses; in the latter, only one response. One can imagine a system in which response preparation could selectively activate a pair of responses without too much danger that either one would gain enough activation to be executed. The two partially activated responses could keep each other in check by mutual inhibition until diagnostic information became available. It might be much more difficult to partially activate a single response, as was necessary in the twochoice task, without activating that response sufficiently to cause it to be made. Further research will be needed to determine whether this is indeed a critical determinant of whether preliminary information is used to prepare responses.

\section{References}

Anzola, G. P., Bertoloni, G., Buchtel, H. A., \& Rizzolatti, G. (1977). Spatial compatibility and anatomical factors in simple and choice reaction time. Neuropsychologia, 15, 295-302.

Coles, M. G. H., Gratton, G., Bashore, T. R., Eriksen, C. W., \& Donchin, E. (1985). A psychophysiological investigation of the continuous flow model of human information processing. Journal of Experimental Psychology: Human Perception and Performance, 11, 529553.

Eriksen, C. W., \& Schultz, D. W. (1979). Information processing in vi- sual search: A continuous flow conception and experimental results. Perception \& Psychophysics, 25, 249-263.

Keppel, G. (1973). Design and analysis: A researcher's handbook. Englewood Cliffs, NJ: Prentice-Hall.

Klapp, S. T., Greim, D. M., Mendicino, C. M., \& Koenig, R. S. (1979). Anatomic and environmental dimensions of stimulus-response compatibility: Implication for theories of memory coding. Acta Psychologica, 43, 367-379.

McClelland, J. L. (1979). On the time relations of mental processes: A framework for analyzing processes in cascade. Psychological Review, 86, 287-330.

Meyer, D. E., Yantis, S., Osman, A. M., \& Smith, J. E. K. (1984). Discrete versus continuous models of response preparation: A reactiontime analysis. In S. Kornblum \& J. Requin (Eds.), Preparatory states and processes (pp. 69-94). Hillsdale, NJ: Erlbaum.

Meyer, D. E., Yantis, S., Osman, A. M., \& Smith, J. E. K. (1985). Temporal properties of human information processing: Tests of discrete versus continuous models. Cognitive Psychology, 17, 445-518.

Miller, J. O. (1982). Discrete versus continuous stage modeis of human information processing: In search of partial output. Journal of Experimental Psychology: Human Perception and Performance, 8, 273296.

Miller, J. O. (1983). Can response preparation begin before stimulus recognition finishes? Journal of Experimental Psychology: Human Perception and Performance, 9, 161-182.

Miller, J. O. (1985a). Discrete and continuous models of divided attention. In M. I. Posner \& O. S. M. Marin (Eds.), Mechanisms of attention: Attention \& performance XI (pp. 513-528). Hillsdale, NJ: Erlbaum.

Miller, J. O. (1985b). A hand advantage in preparation of simple keypress responses: Reply to Reeve and Proctor (1984). Journal of Experimental Psychology: Human Perception and Performance, II, 221233.

Proctor, R. W., \& Reeve, T. G. (1985). Compatibility effects in the assignment of symbolic stimuli to discrete finger responses. Journal of Experimental Psychology: Human Perception and Performance, 11, 623-639.

Proctor, R. W., \& Reeve, T. G. (1986). A caution regarding use of the hint procedure to determine whether partial stimulus information activates responses. Perc 'otion \& Psychophysics, 40, 110-118.

Reeve, T. G., \& Proctor, R 'N. (1984). On the advance preparation of discrete finger responses. Journal of Experimental Psychology: Human Perception and Performance, 10, 541-553.

Reeve, T. G., \& Proctor, R. W. (1985). Nonmotoric translation processes in the preparation of discrete finger responses: A rebuttal of Miller's (1985) analysis. Joumal of Experimental Psychology: Human Perception and Performance, $11,234-241$.

Rosenbaum, D. A. (1980). Human movement initiation: Specification of arm, direction, and extent. Journal of Experimental Psychology: General, 109, 444-474.

Sternberg, S. (1969). Memory scanning: Mental processes revealed by reaction-time experiments. American Scientist, 57, 421-457.

Received November 10, 1986

Revision received February 27, 1987

Accepted March 2, 1987 . 